\title{
Review: no single physical examination sign rules in or out osteoporosis or spinal fracture
}

Green AD, Colón-Emeric CS, Bastian L, et al. Does this woman have osteoporosis? JAMA 2004;292:2890-900

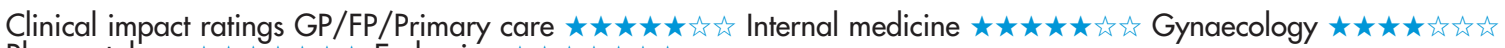

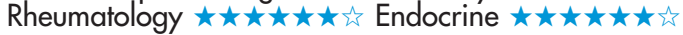

In patients with osteoporosis, what is the accuracy of physical examination signs for diagnosing osteoporosis or spinal fracture?

\section{METHODS}

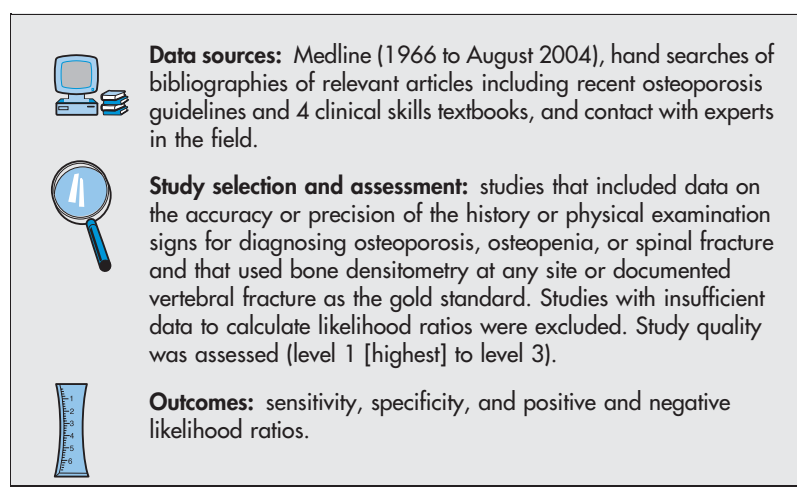

\section{MAIN RESULTS}

14 studies ( 13815 patients) met the selection criteria. All but 1 study included women (mean age range $51-80 \mathrm{y}$ ). 11 studies $(79 \%)$ had level 1 criteria, and 3 studies had level 3 criteria. No single physical examination sign ruled in osteoporosis or spinal fracture without needing further testing. Osteoporosis was best detected by weight $<51 \mathrm{~kg}$, kyphosis, self reported humped back, or $<20$ teeth; and spinal fracture was best detected by wall-occiput distance $>0 \mathrm{~cm}$, and rib-pelvis distance $\leqslant 2$ finger breadths (table). Individual studies that investigated such physical examination signs as height loss, armspan-height difference, grip strength, and hand skinfold thickness had variable or inconclusive results for detecting osteoporosis.

\section{CONCLUSIONS}

The most useful physical examination signs for detecting osteoporosis were weight $<51 \mathrm{~kg}$, kyphosis, self-reported humped back, and $<20$ teeth. Wall-occiput distance $>0 \mathrm{~cm}$ and rib-pelvis distance $\leqslant 2$

For correspondence: Dr C S Colón-Emeric, Duke University Medical Center, Durham, NC, USA. Colon001@mc.duke.edu

Sources of funding: Bureau of Health Professions; National Institutes of Health; VA Medical Research Service. finger breadths were the most useful physical examination signs for detecting spinal fractures.

Commentary

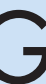
reen ef al have tried to evaluate the accuracy of physical examination signs for diagnosing osteoporosis or spinal fracture. The authors concluded that no single manoeuvre is sufficient to rule out osteoporosis or spinal fractures without further testing. In patients who do not meet current bone mineral density (BMD) screening recommendations, several convenient examination findings (especially low weight) can substantially change the pretest probability of osteoporosis and suggest the need for earlier screening. Wall-occiput distance $>0 \mathrm{~cm}$ and rib-pelvis distance $<2$ finger breadths suggest the presence of occult spinal fractures.

From an evidence-based perspective, none of these manoeurres can, as a single manoeuvre, rule out osteoporosis or spinal fractures. The authors also provide the prevalence of osteoporosis and vertebral fractures for these physical examination findings, which is an important addition to relative risk estimates. More focus should be shifted to prevalence or the absolute risk of a fracture. It would be interesting to find out whether a combination of clinical risk factors and physical examination signs could enhance the predictive ability.

Other attempts to identify patients at high risk of osteoporosis or a high absolute risk of fractures include asking simple questions. For example, have glucocorticoids ever been used? Does the patient smoke, drink, have a history of fracture, or have a family history of fracture or secondary osteoporosis? In addition, existing tools such as the osteoporosis risk assessment instrument (ORAl) in the CaMos study can be used to identify women with low BMD and to predict osteoporosis based on clinical risk factors. ${ }^{1}$ Although the sensitivity of ORAl was high $(93 \%)$, the specificity was low $(46 \%)$, which is expected with most risk scores. All these can be used separately or together with physical examination signs to more accurately predict the absolute fracture risk for a patient and to determine an intervention or the need for a BMD measurement.

Olof Johnell, MD, PhD Malmö University Hospital Malmö, Sweden

1 Cadarette SM, Jaglal SB, Kreiger N, et al. Development and validation of the Osteoporosis Risk Assessment Instrument to facilitate selection of women for bone densitometry. CMAJ 2000;162:1289-94.

Physical examination signs for detecting osteoporosis or spinal fracture in postmenopausal women*

\begin{tabular}{llllll}
\hline Signs for osteoporosis & Number of studies $(\mathbf{n})$ & Sensitivity & Specificity & +LR (95\% CI) & - LR (CI) \\
\hline Weight $<51 \mathrm{~kg}$ & $1(1873)$ & $22 \%$ & $97 \%$ & $7.3(5.0$ to 10.8$)$ & $0.8((0.7$ to 0.9$)$ \\
Self reported humped back & $1(2577)$ & $20.6 \%$ & $97 \%$ & $3.0(2.2$ to 4.1$)$ & $0.85(0.8$ to 0.9$)$ \\
Kyphosis & $1(610)$ & $25 \%$ & $92 \%$ & $3.1(1.8$ to 5.3$)$ & $0.8(0.7$ to 1.0$)$ \\
$<20$ teeth & $1(190)$ & $27 \%$ & $92 \%$ & $3.4(1.4$ to 8.0$)$ & $0.8(0.6$ to 1.0$)$ \\
\hline Signs for spinal fracture & & & & & \\
\hline Wall-occiput distance $>0 \mathrm{~cm}$ & $1(216)$ & $60 \%$ & $87 \%$ & $4.6(2.9$ to 7.3$)$ & $0.5(0.3$ to 0.6$)$ \\
Rib-pelvis distance $\leqslant 2$ finger breadths & $1(781)$ & $88 \%$ & $46 \%$ & $3.8(2.9$ to 5.1$)$ & $0.6(0.5$ to 0.7$)$ \\
\hline *Diagnostic terms defined in glossary. & & & & &
\end{tabular}

